\title{
Trastorno del comedor compulsivo: Incorporación del concepto en la salud pública chilena
}

\author{
Binge eating disorder: \\ Incorporating the concept in the public health system in Chile
}

\begin{abstract}
A literature review on Binge Eating Disorder which is a recently recognized as such by the Diagnostic and Statistical Manual of Mental Disorders versión $\mathrm{V}$, which identifies it as eating disorder pathology that is characterized by an excessive consumption of food with loss of control and compensation methods, causing the person to have excess weight and simultaneously can lead to obesity associated diseases such as diabetes mellitus and hypertension, among others. There is an abundance of worldwide literature on binge eating disorder, its concept, risk factors, characteristics and possible treatments. In Chile there are no studies or strategies to address this disorder that could be present in our population. The purpose of this update is to promote and address the concept of binge eating disorder in the Chilean public health system.
\end{abstract}

Key words: binge eating disorder, obesity, risk factors, characteristics.

\section{INTRODUCCIÓN}

La Organización Mundial de la Salud define obesidad a la acumulación anormal o excesiva de grasa que puede ser perjudicial para la salud (1). La obesidad se ha considerado una situación de importancia por su fuerte asociación con las principales enfermedades crónicas no transmisibles, tales como las enfermedades cardiovasculares, diabetes tipo 2, hipertensión arterial, enfermedades osteoarticulares y algunos cánceres (2). En la salud pública chilena existen protocolos de atención para contrarrestar el exceso de peso, tales como la consulta nutricional en atención primaria de salud que se apoya en los manuales técnicos nutricionales. Además han existido varias estrategias para disminuir esta tendencia, como: Vida Chile, Programa Promoción de la Salud, Escuelas Ego, Elige vivir sano, entre otros (3), todas con una visión de mejorar la calidad de vida a través del fomento de la salud (alimentación saludable, actividad física, ambientes libre de humo de tabaco).

Sin embargo, no se ha considerado la alternativa de que existan trastornos alimentarios que pueden estar en comorbilidad con este estado nutricional.

Los trastornos de conducta alimentaria (TCA) son problemas psiquiátricos, que se definen como una alteración persistente del comer o de conductas relacionadas con esta acción, que se traducen en una alteración en el consumo o absorción de los alimentos, que alteran en forma significativa la salud física o el funcionamiento psicosocial, y que no son secundarios a ningún trastorno médico general u otro síndrome psiquiátrico (4). Cuando se habla de trastorno alimentario se
Fabiola Godoy L.

Unidad de Nutrición, CESFAM Dr. Cristóbal Sáenz Cerda, Lautaro. Chile

Dirigir la correspondencia a: Profesora Fabiola Godoy Leal CESFAM Dr. Cristóbal Sáenz Cerda Lautaro, Chile

Teléfono: (56-45) 2996572 E-mail: fabiolagodoyleal@gmail.com

Este trabajo fue recibido el 21 de Julio de 2015 y aceptado para ser publicado el 30 de Septiembre de 2015. asocia rápidamente a lo más conocidos: anorexia y bulimia, las cuales son patologías psiquiátricas asociadas a la mala alimentación y alteración de la percepción de la imagen corporal (5). Ambos trastornos han sido abordados por los equipos de la salud en Chile, se conoce sus características, los factores de riesgo e incluso los tratamientos, además han sido de objeto de un número considerable de estudios.

Las personas que padecen anorexia, poseen delgadez extrema, en la bulimia poseen un estado nutricional enflaquecido, normal o sobrepeso, según sea la situación; sin embargo existe un trastorno alimentario que puede estar relacionado con el aumento de peso corporal y lo podemos observar en personas con obesidad. Este trastorno se denomina en inglés Binge Eating Disorder y en español Trastorno del Comedor Compulsivo.

Definición de Trastorno del Comedor Compulsivo

El término de Binge Eating (Comedor Compulsivo) fue descrito por primera vez por A. J. Stunkard en la década del 50 (6), el cual lo definió como la ingesta excesiva de alimentos en intervalos de tiempos irregulares. En el año 1994 el Diagnostic and Statistical Manual of Mental Disorder (5) lo incluyó dentro de la clasificación en Desórdenes Alimentarios no Especificados, el cual fue individualizado en la última versión del DSM V, reconociéndolo y declarándolo como patología mental. El Trastorno del Comedor Compulsivo (TCC), se define como la ingesta de grandes cantidades de comida asociado con indicadores subjetivos y comportamiento de 
pérdida de control; angustia significativa por la alimentación compulsiva; y la ausencia del uso regular de comportamientos compensatorios inapropiados: purgaciones, ayunos y ejercicios excesivos (7), esto último es lo que lo diferencia de la Bulimia Nerviosa (8) y por ello se encuentra estrechamente asociado a la obesidad $(9,10)$.

EI DSM, estableció el criterio para identificar a una persona con TCC, la cual es una escala dicotómica en la que debe presentar al menos 3 hallazgos asociados (tabla 1).

\section{Prevalencia}

El TCC no es un trastorno muy conocido en Chile, sin embargo en otros países se han realizado estudios de prevalencia de TCC; uno de ellos afirma que "en Estados Unidos es aproximadamente $2 \%$ en la población en general y $30 \%$ en población de estudio" (11). Otros estudios señalan que en Europa existe una prevalencia menor que la encontrada en Estados Unidos: "Francia posee $0.7 \%$ en la población general y $15.2 \%$ en la población de estudio" (12); Noruega 1.5\% en pruebas basadas en comunidades (13); Inglaterra $0.7 \%$ en pruebas de comunidades de mujeres con historia de obesidad (14); e Italia $12.1 \%$ en población de estudio (15). Investigaciones concluyen que "existe una diferencia de prevalencia, de los TCC, en pruebas clínica en Europa, que posee 10-15\% y Estados Unidos entre 20-30\%" (15), (tabla 2). En Chile sólo existe un estudio en mujeres que nos da un acercamiento a este trastorno, el cual se aplicó los criterios del DSM y nos muestra que sí existe gente con obesidad que posee TCC (16).

\section{Factores de riesgos del TCC}

Existe evidencia científica que identifica los factores de riesgos de las personas que posee dicho trastorno alimentario, siendo los principales las experiencias infantiles adversas, la depresión de los padres, la vulnerabilidad a la obesidad, y la exposición repetida a los comentarios negativos acerca de la forma, el peso y la alimentación. Ciertos rasgos de la infancia y la vulnerabilidad a la obesidad pronunciada los distingue de las personas con bulimia nerviosa (8).

En un estudio realizado con mujeres se observó que tenían un riesgo significativamente mayor a la exposición a ciertos acontecimientos de la vida específica el año antes de que iniciara el trastorno alimentario, por ejemplo: comentarios críticos sobre la forma, el peso, o comer, el estrés relacionado con el trabajo, la escuela u otras fuentes; los principales cambios en las circunstancias de la vida y las relaciones; abuso físico; y sentirse inseguro en una variedad de entornos (17).

EI TCC no se presenta sólo en mujeres sino que también en hombres, así se ha observado en varios estudios en que el hallazgos de encontrar este trastorno no varía de género. (18). EI TCC se asocia de forma fiable con el sobrepeso y la obesidad en las personas que buscan tratamiento. Sin embargo, las personas que poseen obesidad más TCC son diferentes a las personas que sólo tienen obesidad, en comparación con los individuos obesos sin trastorno, aquellos con TCC consumen más calorías en los estudios de laboratorio de la conducta alimentaria, un mayor deterioro funcional y una menor calidad de vida, y de manera significativa mayores niveles de comorbilidad psiquiátrica (19).

\section{Características}

1) Regulación emocional

Se ha planteado la hipótesis de que los individuos con trastornos de la alimentación son vulnerables a involucrarse en "comer en exceso emocional" porque carecen de estrate-

TABLA 1

Escala de Trastorno de Comedor Compulsivo, según DSM V

1. Como más rápido de lo normal

2. Como hasta sentirse muy saciado

3. Ingiero grandes cantidades de comida sin hambre

4. Como sólo por vergüenza

5. Como por disgusto, depresión o culpa por los patrones alimentarios

TABLA 2

Prevalencia de trastorno del comedor compulsivo a nivel mundial

$\begin{array}{ll}\text { Estados Unidos } & \text { 2\% población general y } 30 \% \text { población en estudio } \\ \text { Francia } & 0,7 \% \text { población general y } 15,2 \% \text { población en estudio } \\ \text { Noruega } & 1,5 \% \text { población en general } \\ \text { Inglaterra } & 0,7 \% \text { población de mujeres con historia de obesidad } \\ \text { Italia } & 12,1 \% \text { en población de estudio }\end{array}$

Pruebas clínicas

Europa 
gias y habilidades de regulación emocional de adaptación, incluyendo identificar con claridad y de forma adaptativa frente a los estados emocionales $(20,21)$. Se ha establecido una estrecha relación entre emociones y alimentación, por un lado los estados emocionales y de ánimo pueden influir en la conducta alimentaria, por otro lado, la alimentación puede modificar los estados emocionales y el ánimo (22). La regulación de la emoción puede jugar un papel significativo en el mantenimiento de comer en exceso emocional y la presencia de patologías alimentarias en adultos obesos con TCC (23). Las dificultades con la comida habla del mundo emocional. Son síntomas que avisan de la existencia de conflictos internos y ponen de manifiesto dificultades con la expresión de lo que se siente (24). Cuando no se posee un equilibrio entre emociones y alimentación podemos pasar fácilmente a trastornos de conducta alimentaria. Existe evidencia de que la emoción negativa sirve como detonante de los atracones de comida en las personas que poseen TCC (25). Los atracones de comida están provocadas por una ruptura inmediata de la regulación de la emoción al igual que la bulimia nerviosa, pero se diferencia en que la recuperación del ánimo es más lenta (26).

\section{2) Sobrevaloración del peso}

El modelo cognitivo-conductual de los trastornos alimentarios hace relación a la sobrevaluación del peso refiriéndose al orden superior del contenido cognitivo que refleja las creencias negativas fundamentales sobre uno mismo (por ejemplo, baja autoestima) que puede manifestarse a través de pensamientos automáticos negativos o supuestos disfuncionales respecto a la forma y peso (27).

Existe evidencia que en varios tipos de trastornos alimentarios esta característica está presente. Algunos autores enfatizan que la sobrevaloración del peso debiera ser un criterio de inclusión para detectar pacientes con TCC (28) y otros señalan que no debiera ser considerada como un criterio necesario para el TCC porque excluiría una proporción importante de pacientes, pero si debería ser considerado su dimensión de gravedad (29).

Independiente de que sea o no considerado como criterio diagnóstico, es importante señalar que las personas con TCC poseen una sobrevaloración del peso corporal, adoptando inseguridad, miedos y daño en su autoestima.

\section{3) Ansiedad}

Los trastornos de ansiedad puede ser comprendidos según el DSM V, como episodios persistentes de miedo excesivo o desproporcionado y anticipación de una amenaza futura que va más allá de los períodos de desarrollo apropiados, donde se sobreestima el peligro de las situaciones que temen. Los trastornos de ansiedad difieren uno de otro en los tipos de objetos o situaciones que inducen el miedo, la ansiedad o comportamiento de evitación, y la ideación cognitiva asociada. La ansiedad puede ser comprendida como la contraparte patológica del miedo normal, y se manifiesta como alteraciones del ánimo, de los pensamientos, del comportamiento y de actividades fisiológicas (6). Existen antecedentes en que se asocia a personas con exceso de peso. Cuando la ansiedad sobrepasa determinados límites y empieza a manifestarse una tendencia repetitiva de comer a destiempo, para calmar un estado de malestar, aumentando el aporte calórico (24). En Chile no se han encontrado cifras actualizadas de prevalencia de trastornos de ansiedad en las últimas décadas, sin embargo existe un estudio de prevalencia de patologías psiquiátrica
(ECPP) publicado en el año 2002, que mostró que dentro de los trastornos estudiados el más frecuente corresponde a los desórdenes ansiosos destacándose 17,9\% en la población general (30). Tapia (2006) confirma la asociación positiva entre obesidad y ansiedad, observándose una alta prevalencia de ansiedad en ambos sexos (31), En nuestro país existe un estudio en que se observó la presencia de ansiedad en personas con TCC (16), encontrando que $53.3 \%$ de las mujeres con obesidad y TCC presentaron sintomatología de ansiedad. Otros estudios han analizado la ansiedad social en la cual se sugiere que una mayor ansiedad social y la auto-conciencia elevada se asocian con un mayor trastorno de la alimentación, sobre todo con una mayor preocupación del peso corporal y la frecuencia de comer compulsivamente en personas con TCC (32).

\section{4) Depresión}

Los estados depresivos pueden alterar seriamente la relación con la comida. Las razones de este sufrimiento psíquico, fundamentalmente subjetivo e inconsciente, se traducen en un estado afectivo de intenso dolor que aparece marcado por sentimientos de frustración y fracaso (24). Estudios revelan que pacientes con trastorno de conducta alimentaria presentan alta prevalencia de depresión (33), y a nivel nacional encontramos el hallazgo de que existen personas con TCC con sintomatología depresiva (16). La prevalencia nacional de síntomas depresivo es $17,2 \%$, siendo mayor en mujeres $(25,7 \%)$ que en hombres (8,5\%) (3).

En cuanto a la depresión hay personas que aumentan su apetito, porque el trastorno depresivo puede alterar seriamente la relación con la comida. Las ideas de fracaso, inferioridad o culpa por no alcanzar un ideal son capaces de producir depresión. Uno de los motivos que una persona puede utilizar para hacerse autorreproches continuos es intentar adelgazar y no conseguirlo. Hay personas que desean adelgazar, pero enseguida sienten ganas de comer, después de hacerlo sienten que no tienen fuerza de voluntad y que no podrán llegar a ser como quieren porque no tienen el cuerpo que desean, entonces se deprimen (24).

\section{5) Estrés}

Existen estudios experimentales que analizan la conducta de personas con obesidad con TCC y sin TCC frente a un contexto de estrés, en el cual el primer grupo se observó una motivación más fuerte para la conducta de comer, como así también la falta de percepción de saciedad (34).

La asociación entre el estrés percibido y la planificación de comidas al azar sugiere que, durante períodos de estrés, los individuos son menos propensos a planificar sus comidas cuidadosamente; en consecuencia, más propensos a disfrutar de los alimentos que son característicamente altos en grasa (35).

\section{6) Supresión del pensamiento alimentario}

Los estudios preliminares de muestras no clínicas sugieren intentar evitar los pensamientos de los alimentos, denominado supresión del pensamiento alimentario (SPA), el cual se relaciona con una frecuencia alimentaria y relacionada con la consecuencia de un peso no deseado, sobre todo en las personas obesas $(36,37)$.

EI SPA no se asocia con frecuencia de comidas o con índice de masa corporal, pero se asocia significativamente con altos niveles actuales de trastorno alimentario psicopatología y las variables relacionadas con la obesidad, dieta y la forma de comer compulsivamente (38). 


\section{TCC en Chile}

De acuerdo a todos los antecedentes expuestos debemos preguntarnos ¿cuántas personas con obesidad pueden padecer TCC en nuestro país? Según la última Encuesta Nacional de Salud 2009-2010, existe $64.5 \%$ de población en exceso con peso, de ellos encontramos $25.1 \%$ de la población adulta con obesidad y $2,3 \%$ con obesidad mórbida, alcanzando $34.0 \%$ en las mujeres y $20.5 \%$ en los hombres (39), lo que nos muestra una realidad preocupante debido a la alza que se ha observado durante la última década y su tendencia de crecimiento en edades más tempranas (40).

En la atención clínica se puede escuchar que muchas personas con problemas de obesidad relatan que "sienten ansiedad por la comida", por lo que esta frase puede ser tomada como señal de advertencia, de lo que deseamos plantear: la existencia de un trastorno psicoconductual que está generando secundariamente el exceso de peso.

El tratamiento para disminuir la obesidad en APS se enfoca a un manejo alimentario que contempla la evaluación nutricional (IMC, contorno de cintura), cambio de conducta (según etapas de Prochaska), consejería alimentaria, guías alimentarias y promoción de estilos de vida saludable (3). Sin embargo, no existe ningún acercamiento a observar el exceso de peso como consecuencia de un trastorno alimentario, con factores que puedan incidir en este diagnóstico y características que se puedan identificar y modificar con un tratamiento de psicoterapia dentro de las acciones del manejo de la obesidad del equipo multidisciplinario de salud.

En el mundo existen variados estudios que han planteado algunos tratamientos abordando desde el nivel psicosocial, conductal y psicopatológico (41-43). En Chile debemos empezar por incorporar el concepto del TCC desde la salud pública a nivel central, donde es necesario crear estrategias de intervención por profesionales idóneos en el tema, abordándolo con acciones preventivas, promocionales y generar un tratamiento integral con vinculación en el área psicológica, nutricional y médica, protocolizándolo y socializándolo a nivel nacional en los equipos de atención primaria de salud.

\section{CONCLUSIÓN}

EI TCC es una patología asociada, por sus características, a personas con obesidad. En nuestro país las cifras de obesidad aumentan año a año, por lo que debemos comenzar a sospechar que parte de nuestra población podría estar padeciendo este trastorno. Existen factores psicoconductuales que inciden en su aparición y el afrontamiento del contexto en que vive cada persona, las cuales podrían ser modificadas a nivel de salud pública entregando herramientas necesarias a través del Modelo de Salud Familiar. También existen características que se desencadenan o aumentan en la presencia de este trastorno, las cuales podrían de abordarse de manera integral en la atención primaria de salud, cuando la persona es diagnosticada con exceso de peso y no tan sólo tener acceso a una consulta nutricional, sino que también ser abordada por el equipo multidisciplinario, en especial por el Programa de Salud Mental.

El año 2013 el TCC fue reconocido como tal por el DSM $V$, pero antes de aquello existen décadas de estudio de este trastorno para lograr identificarlo como patología mental independiente, existiendo países con avances considerables en este tema. En Chile debemos comenzar a abordar las directrices de este trastorno tanto en la literatura científica como en estrategias a nivel de salud pública.

\section{RESUMEN}

Se presenta una revisión de la literatura sobre el Trastorno del Comedor Compulsivo (en inglés Binge Eating Disorder) el cual es un patología recientemente reconocida como tal por el Diagnostic and Statistical Manual of Mental Disorders versión $\mathrm{V}$, que lo identifica como trastorno alimentario, que se caracteriza por el consumo excesivo de alimentos (atracones) con pérdida del control y sin métodos compensatorios, que genera que la persona que lo padece tenga una malnutrición por exceso y a la vez puede acarrear patologías asociadas a la obesidad como: diabetes mellitus, hipertensión arterial, enfermedades osteomusculares, entre otros. Existe gran cantidad de literatura a nivel mundial sobre el trastorno del comedor compulsivo abordando concepto, factores de riesgos, características e incluso posibles tratamientos, sin embargo en Chile no existen estudios o estrategias para abordar este trastorno que podría estar presente en nuestra población. El objetivo de esta actualización es incentivar y abordar el concepto del trastorno del comedor compulsivo en la salud pública chilena.

Palabras clave: Trastorno comedor compulsivo, obesidad, factores de riesgo, características.

\section{BIBLIOGRAFIA}

1. World Health Organization (WHO). Obesidad y sobrepeso. 2015. http://www.who.int/mediacentre/factsheets/ fs311/es/index.html.

2. Atalah, E. Epidemiología de la obesidad en Chile. Rev Med Clin Condes, 2012; 23(2): 117-23.

3. Ministerio de Salud de Chile (MINSAL). Estrategia de Intervención Nutricional a través del Ciclo Vital. 2010. http://web.minsal.cl/alimentos_nutricion.

4. Walsh, T. Atypical eating disorder in: Eating Disorder and Obesity. The Guilford Press 1995; 24: 135-40.

5. American Psychiatric Association (APA). Diagnostic and Statistical Manual of Mental Disorder- V. Washington D.C., Estados Unidos: Am Psych Assoc. 2013; 5: 189.

6. Stunkard, A. J. Eating patterns and obesity. Psych Quart, 1959; 33: 284-92.

7. Masheb, R. y Grilo, C. Binge eating disorder: A need for additional diagnostic criteria. Comprehen Psychiat. 2000; 41: 159-62.

8. Fairburn, C., Doll, H., Welch, S., Hay, P., Davies, B. y O'Conner, M. Risk factors for binge eating disorder: a community-based, case-control study. Arch Gen Psych. 1998; 55: 425-32.

9. Marcus, M., Moulton M. y Greeno, C. Binge eating onset in obese patients with binge eating disorder. Addictive Behav. 1995; 20(6): 747-55.

10. Hudson, J., Hiripi, E., Pope, H. y Kessler, R. The prevalence and correlates of eating disorders in the National Comorbidity Survey Replication. Biol Psych, 2007; 61(3); 348-58.

11. Spitzer, R., Yanovsky, S., Wadden, T., Wing, R., Marcus, M., Stunkard, A., Devlin, M., Mitchell, J., Hasin, D. Horne, R. Binge eating disorder: its further validation in a multisite study. Internat J Eating Dis. 1993; 13: 137- 53.

12. Basdevant, A., Poullon, M., Lahlou, N., Le Barzic, M., Brillant, M., y Guy-Grand, B. Prevalence of binge eating disorder in different populations of French women. Internat J Eating Dis. 1995; 18: 309-15.

13. Gotestam, K. Agras, W. General population-based epidemiological study on eating disorder in Norway. Internat J Eating Dis. 1995; 18: 119-26. 
14. Robertson, D. y Palmer, R. The prevalence and correlates of binge eating in a British community sample of women with a history of obesity. Internat J Eating Dis, 1997; 22(3): 323-7.

15. Ramacciotti, C., Coli, E., Passaglia, C., Lacorte, M., Pea, E., y Dell'Osso, L. Binge eating disorder and psycopathological features in a clinical simple of obese people in Italy. Psych Res. 2000; 94: 131-8.

16. Godoy, F. Sintomatología de depresión, ansiedad y baja autoestima en mujeres con obesidad con trastorno del comedor compulsivo. Rev Chil Nutr. 2014; 41(3): 260-3.

17. Pike, K., Dohm, F., Striegel-Moore, R., Wilfley, D. y Fairburn, C. A comparison of black and white women with binge eating disorder. Am J Psych, 2001; 158: 1455-60.

18. Grucza, R., Przybeck, T. y Cloninger, C. Prevalence and correlates of binge eating disorder in a community sample. Comprehen Psych. 2007; 48; 124- 31.

19. Terence, W. Treatment of Binge Eating Disorder. Psych Clin North Am. 2011; 34(4): 773-83.

20. Sim, L., y Zeman, J. The contribution of emotion regulation to body dissatisfaction and disordered eating in early adolescents. J Youth Adolescence, 2006; 35:219-28.

21. Wiser, S., y Telch, C. Dialectical behavior therapy for binge-eating disorder. J Clinical Psychol 1999; 55: 755-8.

22. Gibson, E. Emotional influences on food choice: sensory, physiological and psychological pathways. Physiol Behav. 2006; 89(1): 53-61.

23. Gianini, L., White, M. y Masheb, R. Eating pathology, emotion regulation, and emotional overeating in obese adults with binge eating disorder. Eating Behav, 2013; 14: 309-13.

24. Menéndez, I. Alimentación emocional En Random House., Barcelona, España, 2006.

25. Leehr, E., Krohmer, K., Schag, k., Dresler, T., Zipfel, S. y Giela, K. Emotio $n$ regulation model in binge eating disorder and obesity - a systematic review. Neurosc Biobehavioral Rev. 2015; 49: 125-34.

26. Munsch, S., Meyer, A., Quartier, V. y Wilhelm, F. Binge eating in binge eating disorder: A breakdown of emotion regulatory process?. Psych Res. 2012; 195: 118-24.

27. Fairburn, C. Cognitive behavior therapy and eating disorders, The Guilford Press, New York, 2008.

28. Goldschmidt, A., Hilbert, A., Manwaring, J., Wilfley, D., Pike, K., Fairburn, C. y Striegel-Moore, R. The significance of overvaluation of shape and weight in binge eating disorder. Behav Res Ther. 2010; 48: 187-93.

29. Grilo, C., Crosby, R., Masheb, R., White, M., Peterson, C., Wonderlich, S., Engel, S., Crowd, S. y Mitchell, J. Overvaluation of shape and weight in binge eating disorder, bulimia nervosa, and sub-threshold bulimia nervosa. Behav Res Ther. 2009; 47; 692-6.
30. Rioseco, P., Vicente, B., Saldivia, S., Kohn, R., y Torres, S. Prevalencia de desórdenes psiquiátricos en Chile. Rev Méd Chil. 2002; 130(5): 527-36.

31. Tapia, A. Ansiedad, un importante factor a considerar para el adecuado diagnóstico y tratamiento de pacientes con sobrepeso y obesidad. Rev Chil Nutr. 2006; 33: 352-7.

32. Sawaoka, T., Barnes, R., Blomquist, K., Masheb, R. y Grilo, C. Social anxiety and self-consciousness in binge eating disorder: associations with eating disorder psychopathology. Comprehen Psych, 2012; 53: 740-5.

33. Strober M. y Katz J. Do eating disorders and affective disorders share a common etiology? A dissenting opinion. Int J Eat Disord. 1987; 6: 171-80.

34. Schulz, S. y Laessle, R. Stress-induced laboratory eating behavior in obese women with binge eating disorder. Appetite. 2012; 58: 457-61.

35. Sims, R., Gordon, S., Garcia, W., Clark, E., Monye, D., Callender, C. y Campbell, A. Perceived stress and eating behaviors in a community-based simple of African Americans. Eating Behav. 2008; 9: 137-42.

36. Dejonckheere, P., Braet, C., y Soetens, B. Effects of thought suppression on subliminally and supraliminally presented food-related stimuli. Behav Change, 2003; 20: 223-30.

37. Smart, L., y Wegner, D. Covering up what can't be seen: Concealable stigma and mental control. J Personality Social Psychol. 1999; 77: 474-86.

38. Barnes, R., Masheb, R., White, M. y Grilo, C. Examining the relationship between food thought suppression and binge eating disorder. Comprehen Psych. 2013; 54: 1077-81.

39. Ministerio de Salud (MINSAL) Encuesta Nacional de Salud 2009 - 2010. MINSAL, 2010, p. 17.

40. Kain, J., Galván, M., Taibo, M., Corvalán, C., Lera, L. y Uauy, R. Evolución del estado nutricional de niños chilenos desde la etapa preescolar a la escolar: resultados antropométricos según procedencia de las mediciones. Arch Latinoam Nutr. 2010; 60(2): 155-9.

41. Grilo, C., White, M., Gueorguieva, R., Barnes, R. y Masheb, R. Self-help for binge eating disorder in primary care: A randomized controlled trial with ethnically and racially diverse obese patients. Behav Res Ther. 2013; 51: 855-61.

42. Robinson, A. Integrative Response Therapy for Binge Eating Disorder. Cognitive Behav Pract. 2013; 20: 93-105.

43. Grilo, C., Masheb, R., White, M., Gueorguieva, R., Barnes, R., Walsh, B., McKenzie, K., Genao, I. y Garcia, $R$. Treatment of binge eating disorder in racially and ethnically diverse obese patients in primary care: Randomized placebo-controlled clinical trial of self-help and medication. Behav Res Ther. 2014; 58: 1-9. 